\title{
Mechanism for the stabilization of protein clusters above the solubility curve
}

\author{
James F. Lutskd* and Grégoire Nicolis \\ Center for Nonlinear Phenomena and Complex Systems, Code Postal 231, \\ Université Libre de Bruxelles, Blvd. du Triomphe, 1050 Brussels, Belgium
}

(Dated: August 11, 2018)

\begin{abstract}
Pan, Vekilov and Lubchenko[J. Phys. Chem. B 2010, 114, 76207630] have proposed that dense stable protein clusters appearing in weak protein solutions above the solubility curve are composed of protein oligomers. The hypothesis is that a weak solution of oligomer species is unstable with respect to condensation causing the formation of dense, oligomer-rich droplets which are stabilized against growth by the monomer-oligomer reaction. Here, we show that such a combination of processes can be understood using a simple capillary model yielding analytic expressions for the cluster properties which can be used to interpret experimental data. We also construct a microscopic Dynamic Density Functional Theory model and show that it is consistent with the predictions of the capillary model. The viability of the mechanism is thus confirmed and it is shown how the radius of the stable clusters is related to physically interesting quantities such as the monomer-oligomer rate constants.
\end{abstract}

\section{INTRODUCTION}

Proteins in solution demonstrate a surprisingly rich variety of phenomena, many of which have biological implications. These include liquid-liquid separation (e.g. protein rich droplets forming in a weak solution), crystallization and gelation[1]. Perhaps most surprising is the relatively recent discovery of the existence of protein clusters having a typical, stable size and long life time that have been found to exist under a wide variety of conditions including those for which no condensed phases are stable 2 4]. There is abundant evidence that these clusters play an important role in protein crystallization [5-7] and it has been suggested that they play a similar role in the formation of protein aggregates that are actors in pathologies such as hemoglobin polymers in sickle cell anemia and fibrils of misfolded proteins that underlie various neurological disorders $[8]$. Thus, understanding their origin and nature is of importance for both fundamental and practical reasons.

The existence of stable clusters anywhere in the phase diagram is mysterious. A subcritical droplet of a condensed phase should, by definition, evaporate whereas a supercritical droplet should grow until all available material is incorporated. Multiple droplets can compete with one another for the available material slowing down the growth process during the so-called ripening stage, until only very large droplets remain. Coelescence of droplets can also contribute to this outcome. However, the experimental evidence indicates that for the protein clusters, any such ripening ends after a finite time leaving a stable population of droplets much smaller than expected according to classical scenarios [9].

Recently, Vekilov and co-workers proposed that the stable protein clusters might not be composed of the native protein but, rather, of some complex formed from them such as an oligomer or a mis-folded monomer 8 - 10]. Their idea was that in the original, weak protein solution the new species is in equilibrium with the protein monomer but that the phase diagram of the new species is such that a condensed phase is favored so that super-critical clusters (e.g. oligomer-rich droplets) can form. However, since the density of of the secondary species within the clusters would be well above the concentration for chemical equilibrium with the monomer, there would be a tendency for the secondary species to convert back to monomers within the cluster thus impeding its growth. If one adds the assumption that excluded volume effects prevent a high monomer concentration within the droplet (which could also lead to a stable chemical equilibrium between the two species) then a possible mechanism for stabilization is apparent. Although this idea has motivated further experimental work, little has been done to formalize it theoretically. The goal of this paper is to do so at two levels. First, the stabilization problem will be considered phenomenologically using concepts from Classical Nucleation Theory (CNT) [11]. This will result in simple analytic expressions for the size of the stable clusters as functions of the properties of the original solution and of the concentration and pressure of the secondary species. This analytic relation opens the door to the determination of these properties from experiment. Our second contribution is the formulation of a detailed Dynamic Density Functional Theory (DDFT) model based on the same assumptions and used to confirm the phenomenological predictions while providing a more fundamental means of investigating the nature of the clusters.

*jlutsko@ulb.ac.be http://www.lutsko.com 


\section{PHENOMENOLOGY}

For the sake of concreteness, we will assume that the secondary population is composed of dimers. All of the subsequent development can be trivially adapted to other possibilities. We then begin by postulating a simple massbalance reaction model for the conversion of monomers into dimers and vice-versa. Calling the monomer number density $n_{1}$ and the dimer density $n_{2}$ this takes the form

$$
\begin{aligned}
& \frac{d n_{1}}{d t}=-2 k_{1} n_{1}^{2}+2 k_{2} n_{2} \\
& \frac{d n_{2}}{d t}=k_{1} n_{1}^{2}-k_{2} n_{2}
\end{aligned}
$$

where the factors of two ensure that the total number of protein molecules, $n=n_{1}+2 n_{2}$ within any small volume element, is conserved in the absence of spatial inhomogeneities. The square of the monomer density occurs because two monomers must meet to form a dimer. This gives a relation between the equilibrium densities of $k_{1} n_{1}^{(e q) 2}=k_{2} n_{2}^{(e q)}$. The rate equations can be solved exactly and it is found that the non-conserved difference $n_{1}-2 n_{2}$ relaxes exponentially at long times with time constant $\sqrt{k_{2}^{2}+8 n k_{1} k_{2}}$.

Now, let us consider a pure solution of dimers and we assume that conditions are such that the fluid nucleates a dense phase. In the capillary approximation used in CNT, it is assumed that the density inside a cluster having radius $R$ is constant, $n_{2}(r<R)=n_{2}^{(0)}$ and equal to the density of the homogeneous, condensed phase, while the density outside the cluster, $n_{2}(r>R)=n_{2}^{(\infty)}$ is also constant. In this case, the rate of growth of a sufficiently large supercritical cluster is, under the diffusion-limited conditions expected to dominate for macromolecules in solution, given by [12]

$$
\frac{d R}{d t}=a R^{-1}, a=D n_{2}^{(\infty)} \frac{\beta P\left(n_{2}^{(0)}\right)-\beta P\left(n_{2}^{(\infty)}\right)}{\left(n_{2}^{(0)}-n_{2}^{(\infty)}\right)^{2}}
$$

where $D$ is the tracer diffusion constant for a dimer molecule in solution, $P\left(n_{2}\right)$ is the pressure for the dimers at density $n_{2}$ and $\beta=1 / k_{B} T$ with $T$ the temperature and $k_{B}$ Boltzmann's constant.. This gives the classic result $R \sim t^{1 / 2}$.

Now, let us consider the effect of adding monomers to the picture. Outside the cluster, the monomers and dimers will reach equilibrium so we have that $k_{1} n_{1}^{(\infty) 2}=k_{2} n_{2}^{(\infty)}$. We assume that the monomers and dimers have no interaction aside from excluded volume effects. In this case adding monomers to the cluster raises its free energy so that one expects the monomers to be expelled by diffusion leading to the hypothesis that the monomer concentration inside the cluster is very low, $n_{1}^{(0)} \simeq 0$. Clearly, the realization of this condition will depend on diffusion being sufficiently fast compared to the rate of production of the monomers. In terms of the dimer concentration within the cluster, the net effect (conversion of dimers to monomers and expulsion of the monomers) is a simple extinction reaction that lowers the total number of dimers, $N_{2}=\frac{4 \pi}{3} n_{2} R^{3}$, according to $d N_{2} / d t=-k_{2} N_{2}$. Since the free energy of the cluster will be minimized by maintaining a dimer density near that of the thermodynamically stable condensed phase, this leads to a reduction in the size of the cluster given by $d R / d t=-k_{2} R / 3$.

The combined effect of the reaction and of diffusion gives an evolution equation for the radius of the form

$$
\frac{d R}{d t}=a R^{-1}-k_{2} R / 3 .
$$

In this simple relation, the term driving growth scales more weakly than the term opposing growth which is the opposite of what happens in classical nucleation theory. As a consequence, the dynamics are reversed: small clusters tend to grow while large clusters tend to shrink until the cluster reaches a stable, stationary size as is reflected in the exact solution to Eq.(3),

$$
R^{2}(t)=R_{s}^{2}+\left(R^{2}(0)-R_{s}^{2}\right) e^{-\frac{2 k_{2}}{3} t}, \quad R_{s}=\sqrt{\frac{3 a}{k_{2}}} .
$$

These expressions link accessible experimental quantities such as the cluster size and the rate of relaxation of the system to the parameters governing the model. In particular, they in principle give experimental access to the rate constants since one expects the exterior dimer concentration, $n_{2}^{(\infty)}$, to be in equilibrium with the monomer concentration outside the droplet $\left(k_{1} n_{1}^{(\infty) 2}=k_{2} n_{2}^{(\infty)}\right)$ so that measurement of the respective concentrations, together with knowledge of $k_{2}$, allows the determination of $k_{1}$ and thus complete characterization of the reaction between the two species. 


\section{MICROSCOPIC MODEL}

To test these ideas, we now describe a microscopic model that incorporates the growth of a super-critical droplet and the excluded volume interaction of the monomer and dimer species. Our approach is based on Dynamic Density Functional Theory (DDFT) which is commonly used to describe the dynamics of over-damped systems (such as colloids and macromolecules in solution) under conditions such that thermal fluctuations may be ignored [13 15]. In DDFT, the fundamental quantity is the time-dependent local density (or equivalently, concentration) $n(\mathbf{r} ; t)$. The diffusion-limited growth of a super-critical droplet in a pure solution of dimers (i.e. with no monomers present) is governed by

$$
\frac{d n_{2}(\mathbf{r} ; t)}{d t}=D_{2} \nabla \cdot n_{2}(\mathbf{r} ; t) \nabla \frac{\delta F\left[n_{2}\right]}{\delta n_{2}(\mathbf{r} ; t)}
$$

where $D_{2}$ is the tracer diffusion constant for the dimers. The free energy functional will be taken to have the squared-gradient form 15,16$]$

$$
F\left[n_{2}\right]=\int\left\{f_{2}\left(n_{2}(\mathbf{r} ; t)\right)+\frac{1}{2} g_{2}\left(\nabla n_{2}(\mathbf{r} ; t)\right)^{2}\right\} d \mathbf{r}
$$

where $f_{2}\left(n_{2}\right)$ is the Helmholtz free energy per unit volume for a homogeneous fluid at density $n_{2}$ and $g_{2}$ is a constant that can be calculated from the interaction potential[17]. In the following, the dimers will be described generically using a Lennard-Jones interaction potential in which case good parameterizations are available in the literature 18]. In the limit of low densities, the gradient term is negligible and the Helmholtz free energy goes to the ideal gas form $f_{2}\left(n_{2}\right) \rightarrow f^{(i d)}\left(n_{2}\right)=n_{2} \ln n_{2} \Lambda^{3}-n_{2}$ so that the left hand side of the DDFT equation reduces to $D_{2} \nabla^{2} n_{2}$, i.e. it becomes the diffusion equation. Thus, one may think of DDFT as a generalization of the diffusion equation that accounts for particle interactions.

To generalize to two species, the free energy functional is replaced by one depending the local densities of both species, $F\left[n_{1}, n_{2}\right]$, and a second DDFT equation is included for $n_{1}$. In the present case, we must also include the chemical reactions thus giving

$$
\begin{aligned}
& \frac{d n_{1}(\mathbf{r} ; t)}{d t}=D_{1} \nabla \cdot n_{1}(\mathbf{r} ; t) \nabla \frac{\delta F\left[n_{1}, n_{2}\right]}{\delta n_{1}(\mathbf{r} ; t)}-2 k_{1} n_{1}(\mathbf{r} ; t)^{2}+2 k_{2} n_{2}(\mathbf{r} ; t) \\
& \frac{d n_{2}(\mathbf{r} ; t)}{d t}=D_{2} \nabla \cdot n_{2}(\mathbf{r} ; t) \nabla \frac{\delta F\left[n_{1}, n_{2}\right]}{\delta n_{2}(\mathbf{r} ; t)}+k_{1} n_{1}(\mathbf{r} ; t)^{2}-k_{2} n_{2}(\mathbf{r} ; t)
\end{aligned}
$$

In principle, for a non-ideal system we should replace the concentrations occurring in the chemical reaction terms by the corresponding activities. Here, we keep the simple form given above for the sake of comparison to the phenomenological model and defer further discussion of this point to the Conclusions.

Finally, the form of the free energy functional must be specified. Since the monomers are supposed to be above their critical point, we simply treat them as hard spheres with hard-sphere diameter $d$ so as to account for excluded volume effects. The final form we employ is

$$
\begin{aligned}
F\left[n_{1}, n_{2}\right] & =\int\left\{f_{h s}\left(n_{1}(\mathbf{r} ; t) ; d\right)+\frac{1}{2} g_{1}\left(\nabla n_{1}(\mathbf{r} ; t)\right)^{2}\right\} d \mathbf{r} \\
& +\int\left\{f_{L J}\left(n_{2}(\mathbf{r} ; t)\right)+\frac{1}{2} g_{2}\left(\nabla n_{2}(\mathbf{r} ; t)\right)^{2}\right\} d \mathbf{r} \\
& +\int\left\{f_{h s}^{(e x)}\left(n_{1}(\mathbf{r} ; t)+n_{2}(\mathbf{r} ; t) ; d\right)-f_{h s}^{(e x)}\left(n_{1}(\mathbf{r} ; t) ; d\right)-f_{h s}^{(e x)}\left(n_{2}(\mathbf{r} ; t) ; d\right)\right\} d \mathbf{r}
\end{aligned}
$$

The third line accounts for the mutual excluded volume interaction of the two species: we treat both as hard spheres of diameter $d$ and replace their individual hard-sphere contributions to the excess free energy by one dependent on the sum of the local densities. (Note that the excess part of the free energy is just $f^{(e x)}=f-f^{(i d)}$ : we only replace the excess part because the ideal contributions are already accounted for.) If either density is zero, this interaction term vanishes. Of course, a dimer with twice the mass of a monomer and the same density would have a diameter about $25 \%$ larger but for simplicity we ignore this relatively small difference. Similarly, we take $g_{1}=g_{2}=g_{L J}$ and $D_{1}=D_{2}$ since we expect the differences in these coefficients to be of no physical importance. A final simplification is that we do not include a cross term involving the gradients. This model is a generalization of the model used by Huberman to discuss the appearance of striations in a reacting system[19]. Huberman's model was constructed 


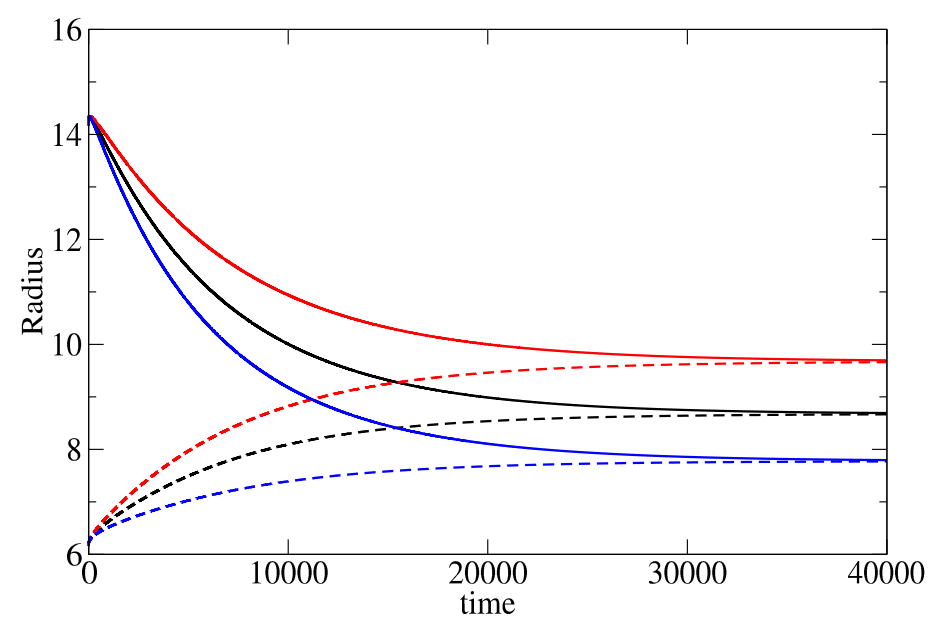

FIG. 1. Behavior of the cluster radius as a function of time (both in dimensionless units) for three different values of the reaction parameter, $k_{1}^{*}=8.75 \times 10^{-4}$ (upper curve), $7.5 \times 10^{-4}$ (middle curve) and $10^{-3}$ (lower curve). In each case, two initial configurations are used: one with a small initial displacement of the critical cluster, and one with a large initial displacement. In all three cases, both initial conditions lead to the same final cluster radius thus demonstrating the stability of the final cluster.

in the approximation of a single active reactant with an autocatalytic chemical reaction out of equilibrium. Here, the presence of two species participating in an equilibrium reaction is fully accounted for. This necessarily requires adding an additional contribution to the free energy and, most importantly, the third line in Eq.(8) which accounts for the most basic excluded-volume interaction of the two species. Note that in this setting the conservation condition $n_{1}+2 n_{2}=$ const. no longer holds locally.

The Lennard-Jones potential introduces a length scale, $\sigma$, and an energy scale $\varepsilon$. In the following, temperature will be reported in the scaled units $T^{*}=k_{B} T / \varepsilon$ and all lengths will be scaled by $\sigma$. We also take the hard-sphere diameter $d=\sigma$ : typical prescriptions such as Barker-Henderson [20] change this by a few percent but for present purposes this difference is unimportant. A time scale, $\tau$, is introduced such that $D_{2} \tau / \sigma^{2}=1$. After scaling, the available parameters are the monomer background density, the dimer supersaturation, the scaled temperature and the scaled reaction coefficient $k_{1}^{*}$. The dimer reaction constant is determined via the equilibrium condition $k_{1}^{*} n_{1}^{(\infty) * 2}=k_{2}^{*} n_{2}^{(\infty) *}$. We report results here for $T^{*}=0.8$ and supersaturation $n_{2}^{(\infty)} / n_{2}^{(\operatorname{coex} x)}=2$ where $n_{2}^{(\operatorname{coex})}$ is the vapor density at coexistence at this temperature. Under these conditions the density in the vapor is $n_{2}^{(\infty) *}=0.012$ and in the condensed phase $n_{2}^{*}=0.85$. The background monomer density is taken to be 5 times that of the dimer phase. In reality, this ratio is thought to be much greater [8] but the computational cost of the calculation increases with this ratio so our choice represents a compromise. The only remaining parameter is $k_{1}^{*}$ which is discussed below.

Our calculations were performed assuming spherical symmetry with boundary conditions appropriate for an open system (see Supplementary Material for technical details). We began by locating the critical cluster for the pure dimer system. With the chosen parameters, this has radius $R_{c}^{*}=5.2$. We then make this supercritical by increasing its radius an amount $\Delta R$ and then adding in the monomers. Further details are given in the Supplementary Material as are details of the numerical algorithms used to integrate the DDFT equations. Also discussed there are the question of the definition of the radius to use for comparing the capillary model to the DDFT and the agreement between the two theories for the case of the growth of a super-critical droplet in a single-component system.

The evolution of the cluster radius for three different values of the reaction constant is shown in Fig. 1. In each case, two initial displacements are used: an "under" displacement of one unit (broken lines) and an "over" displacement of 9 units (full lines). The fact that the under- and over-displaced clusters evolve to the same final cluster is strong empirical evidence for the stability of the final cluster. The structure of the stable cluster is shown in Fig. 22 where it can be seen that most of the monomer species is expelled from the cluster except in the interfacial region.

The scaling relation between the stable radius and the reaction constant $k_{2}^{*}$ predicted by the capillary model, Eq.(44), is tested against the numerical DFT results in Fig. 3. For lower values of the dimensionless reaction coordinate, there are significant deviations as is to be expected since the capillary model is only accurate for large clusters. As the 


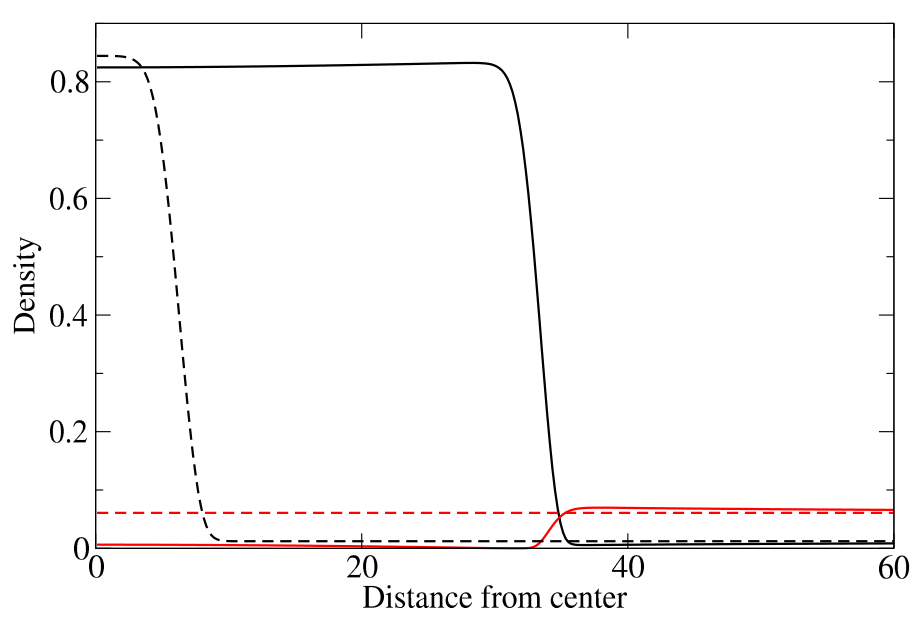

FIG. 2. Structure of the stable cluster for $k_{1}^{*}=7.5 \times 10^{-5}$. The density (concentration) of the monomer species (solid red line) and the dimer species (solid black line) is shown as functions of distance from the center of the cluster. The initial condition is also shown using dashed-lines.

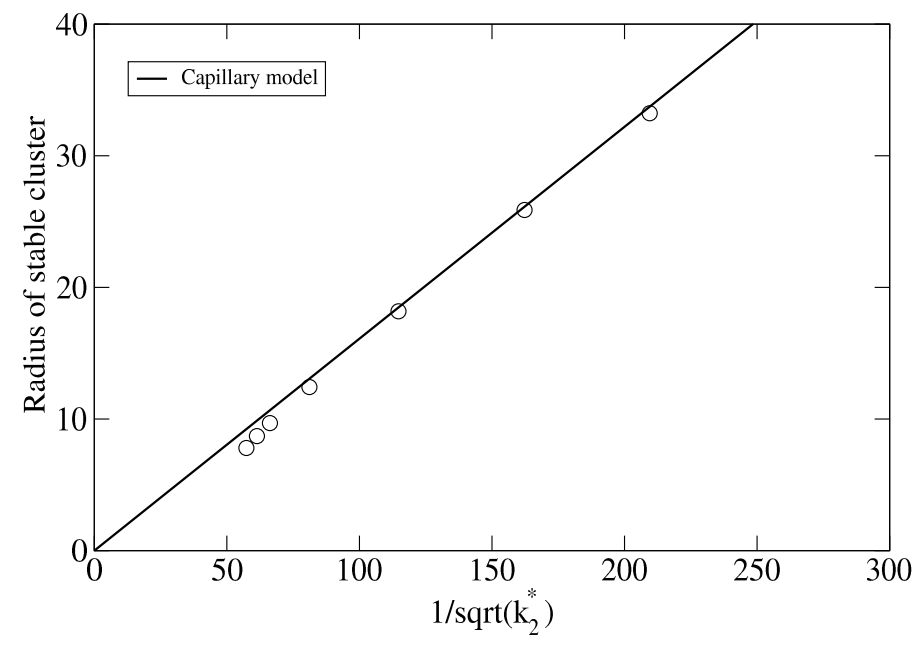

FIG. 3. Predicted stable radius from the capillary model, Eq.(4), compared to the results of numerical integration of the DFT model (symbols) as a function of $1 / \sqrt{k_{2}^{*}}$. With these variables, the prediction is simply a straight line.

reaction rate decreases, and the size of the stable cluster increases, convergence to the prediction is evident.

\section{CONCLUSIONS}

We have shown that super-critical clusters, which would otherwise be unstable with respect to growth, can be stabilized by means of the combined effect of diffusion and a chemical reaction. Diffusion - driven by thermodynamics - leads to the purification of the cluster so as to lower its free energy. The purified cluster is then in turn subject to degradation due to the conversion of the dimer species to monomers. This dynamic process can be successfully described by a simple capillary model as well as more systematically investigated by means of a microscopic Dynamic 
Density Functional Theory model. The two approaches were shown to be in agreement. While these results were necessarily achieved for specific choices of molecular interactions and, particularly, for a specific chemical reaction, it is clear that the arguments may be trivially adapted to other choices.

The microscopic DDFT model presented here is a natural generalization of the standard reaction-diffusion model used to describe chemical reactions in spatially extended systems. The crucial element in our formulation of this generalization is the free energy functional and particularly the interaction term given in the third line of Eq. (8). The free energy contribution can be viewed as giving rise to a density-dependent diffusion constant which, for the condensed phase, is negative thus driving growth of a cluster rather than its diffusive evaporation as in ordinary diffusion. The interaction term in the free energy is critical in that it leads to a monomer diffusion constant that increases with increasing density of dimers thus causing expulsion of the monomers from the dimer cluster. This leads to a locally frozen nonequilibrium steady state in which a current of dimers flows into the cluster where they are converted to monomers and expelled in the form of a corresponding outward current. In this state growth of the droplet and the conversion of species are mutually quenched. Since such a nonequilibrium state cannot persist indefinitely without a driving force (due e.g., to mode-coupling effects not considered in the over-damped limit used here[12]), the clusters are not expected to be stable indefinitely. Furthermore, shape fluctuations are also likely to prove destabilizing since any deviation from a spherical shape will lower the thermodynamic driving force for growth and potentially lead to irreversible shrinking of the cluster to a size below the critical radius. Finally, as mentioned already earlier, in the results presented here the system is actually treated as an open system spatially infinite, continually replenished by monomers and coupled implicitly to an infinite solvent 12] that acts as a reservoir. This further postpones the establishment of a global equilibrium throughout.

We conclude with several observations concerning this mechanism. First, there is no constraint on the free energy of the stable droplet since the only requirement is that it be larger than the critical cluster. It could therefore have a free energy nearly as high as that of the critical cluster (leading to a relatively low number of such droplets in equilibrium) or it could have an arbitrarily low free energy (leading to a large population).

Second, we have assumed that when the reaction removes dimers the density of the cluster remains constant so that the net effect is that the cluster shrinks in size. This only makes physical sense if the reaction is in some sense slow compared to the process of removing monomers from the cluster (i.e. diffusion). Were this not the case, monomers would quickly build up within the cluster and poison it leading to its collapse.

Third, we note the generality of the mechanism leading to a stable cluster with a characteristic size: a force driving growth that scales more slowly than a force opposing growth. Regardless of the mechanisms giving rise to the forces, these are the required elements. Clusters in other systems could be stabilized by some other combination of growth-promoting and -opposing forces provided the relative scaling satisfies this rule.

Fourth, one can contrast this mechanism to that stabilizing vesicles. The latter consist of a volume with amphiphilic molecules arranged on its surface so that their hydrophobic parts are inside the volume, shielded from water, while their hydrophilic parts are on the outside of the surface, exposed to the water. Within the vesicle could be void, more of the apmphiphilic molecules or some other substance. If the interior has a higher free energy than the solution, the vesicle can be stabilized in the same manner as proposed above: the surface dominates the free energy of small vesicles leading to growth while the volume dominates large vesicles leading to dissolution. However, in the case of vesicles there is another factor: such a system can increase its surface to volume ratio, and hence decrease its free energy, by becoming non-spherical ( by becoming flat, in the extreme limit). In our case, the free energy is minimized by a spherical shape so that the mechanism favors the formation of spherical clusters.

Fifth, there is no scope within this model for ripening: i.e. the growth of larger clusters at the expense of smaller ones. Something like ripening has in fact been reported by $\mathrm{Li}$ et al. [9] albeit with the unusual feature that the ripening stops while there is still a finite population of clusters. If the present model were correct, this "ripening" would have to be reinterpreted: perhaps as a slowly relaxing transient. As stated above, the reaction must be slow compared to diffusion, as is reflected in the small dimensionless reaction constants used in our work, and this could simply result in very slow dynamics for the entire system. Alternatively, it is possible that the dimer to monomer reaction is suppressed within the cluster (due to the high free energy barrier involved in removing a dimer from the condensed phase) and that the reaction is most productive only in the boundary of the cluster (where the dimer is in an energetically unfavorable state). In this case, the reaction term in Eq.(3) would be a constant rather than scaling as $R$ (in fact, $R$ would be replaced by the characteristic width of the boundary region) and this would lead to algebraic rather than exponential relaxation of the cluster to its stable size. To capture this, the model could be modified by replacing the concentrations in the rate equations with more general expressions involving the chemical affinities. Such an algebraic dynamics combined with small reaction constants could well give transients that decay very slowly and could therefore be interpreted as a transient ripening.

This is related to our sixth and final point. We mentioned above that for consistency, we should replace the concentrations appearing in the chemical reaction kinetics by the corresponding activities, $n_{i}(r, t) \rightarrow n_{i}^{(0)} \exp \left(\beta \mu_{i}(r, t)-\beta \mu_{i}\right)$ where the local chemical potential is $\mu_{i}(r, t)=\frac{\delta F}{\delta n_{i}(r, t)}$ and where $\mu_{i}$ is the chemical potential for species $i$ in the homo- 
geneous system in which $n_{i}(r, t)=n_{i}^{(0)}$. This has not been used in the present work in order to explore the consistency of the simple capillary model with the microscopic model in the case that the relation between the two is most straightforward. We speculate that the effect of the use of the activities will be a suppression of the dimer to monomer reaction within the cluster and an enhancement of the importance of the reaction in the interfacial region, therefore possibly leading to the scenario alluded to above of an algebraic rather than exponential relaxation. Preliminary calculations using the activities supports this and the issue will be discussed more fully in a future publication.

\section{ACKNOWLEDGMENTS}

This work was supported in part by the European Space Agency under contract number ESA AO-2004-070. The authors thank Peter Vekilov for numerous conversations on this subject and Pierre Gaspard for useful comments concerning the model.

[1] J. D. Gunton, A. Shiryayev, and D. L. Pagan, Protein Condensation: Kinetic Pathways to Crystallization and Disease (Cambridge University Press, Cambridge, 2007) p. 376.

[2] W. Pan, O. Galkin, L. Fibobelo, R. L. Nagel, and P. G. Vekilov, Biophys. J. 92, 267 (2007).

[3] O. Gliko, N. Neumaier, W. Pan, I. Haase, M. Fischer, A. Bacher, S. Weinkauf, and P. G. Vekilov, Journal of the American Chemical Society 127, 3433 (2005).

[4] O. Gliko, W. Pan, P. Katsonis, N. Neumaier, O. Galkin, S. Weinkauf, and P. G. Vekilov, The Journal of Physical Chemistry B 111, 3106 (2007).

[5] P. G. Vekilov, Crys. Growth and Design 4, 671 (2004).

[6] J. F. Lutsko and G. Nicolis, Phys. Rev. Lett. 96, 046102 (2006).

[7] M. Sleutel and A. E. S. V. Driessche, Proc. Nat. Acad. Sci. 111, E546 (2014).

[8] W. Pan, P. G. Vekilov, and V. Lubchenko, J. Phys. Chem. B 114, 7620 (2010).

[9] Y. Li, V. Lubchenko, M. A. Vorontsova, L. Filobelo, and P. G. Vekilov, J. Phys. Chem. B 116, 10657 (2012).

[10] Y. Li, V. Lubchenko, and P. G. Vekilov, Rev. of Sci. Instruments 82, 053106 (2011).

[11] D. Kashchiev, Nucleation : basic theory with applications (Butterworth-Heinemann, Oxford, 2000).

[12] J. F. Lutsko, J. Chem. Phys. 136, 034509 (2012).

[13] U. M. B. Marconi and P. Tarazona, J. Chem. Phys. 110, 8032 (1999).

[14] A. J. Archer and R. Evans, J. Chem. Phys. 121, 4246 (2004).

[15] J. F. Lutsko, Adv. Chem. Phys. 144 (2010).

[16] R. Evans, Adv. Phys. 28, 143 (1979).

[17] J. F. Lutsko, J. Chem. Phys. 134, 164501 (2011).

[18] J. K. Johnson, J. A. Zollweg, and K. E. Gubbins, Mol. Phys. 78, 591 (1993).

[19] B. A. Huberman, J. Chem. Phys. 65, 2013 (1976).

[20] J. A. Barker and D. Henderson, J. Chem. Phys. 47, 4714 (1967). 